\title{
Atomic Force Microscopy Studies on GaAs/In Bilayers Deposited on Si (100)
}

\author{
Miguel Ángel Venegas', Roberto Bernal-Correa², Máximo López-López¹, \\ Álvaro Pulzara-Mora ${ }^{2}$ \\ ${ }^{1}$ Departamento de Física, Centro de Investigación y de Estudios Avanzados del I.P.N., México D.F., México \\ ${ }^{2}$ Laboratorio de Nanoestructuras Semiconductoras, Universidad Nacional de Colombia, Manizales, Colombia \\ Email: aopulzaram@unal.edu.co
}

Received 5 January 2015; accepted 21 January 2015; published 30 January 2015

Copyright (C) 2015 by authors and Scientific Research Publishing Inc.

This work is licensed under the Creative Commons Attribution International License (CC BY). http://creativecommons.org/licenses/by/4.0/

(c) (i) Open Access

\begin{abstract}
GaAs/In bilayers were prepared by RF Magnetron Sputtering in an Ar atmosphere on Si (100) substrates using high purity $(95.95 \%)$ GaAs $(100)$ and In targets. The growth temperatures were $300^{\circ} \mathrm{C}$ and $580^{\circ} \mathrm{C}$ for the high purity targets of In and GaAs, respectively. Three samples were prepared: the deposition time $\left(t_{d}\right)$ for the GaAs layers was fixed to 30 minutes, while varied for the In layers from $t_{d}=10,15$, and 20 minutes. The morphological and optical studies of the samples were made by means of Amplitude Modulation Atomic Force Microscopy (AM-AFM). In order to analyze and correlate surface morphology and alloy composition properties, the as-prepared samples were cleaved along the [001] direction and subsequently studied by AM-AFM-micrographs. From topographic images, a statistical study of the roughness and grain size was made. Additionally, cross sectional AM-AFM-micrographs were performed for each sample, where the phase channel, which is sensitive to the material properties of the specimen, was of particular interest.
\end{abstract}

\section{Keywords}

InGaAs, Magnetron Sputtering, AFM

\section{Introduction}

Indium Gallium Arsenide (InGaAs) heterostructures, often regarded as GaAs and InAs binary alloys, are a III-V semiconductor compound largely used in the electronic and optoelectronic industry. The range of practical devices fabricated with this compound, grown on $\mathrm{Si}$ (100) substrates, due to the different band gaps related in the system ( $0.33 \mathrm{eV}$ for InAs, $1.43 \mathrm{eV}$ for $\mathrm{GaAs}$ and $1.12 \mathrm{eV}$ for $\mathrm{Si}$ ), varies from optical sensors, photodiodes, solar cells and many other kind of devices [1] [2]. 
Among the several techniques and methods used to growth InGaAs compounds, the Radio Frequency Magnetron Sputtering technique is of special interest, due to its low cost fabrication process compared with other growing techniques. Characterization, understanding and controlling the growing process is necessary in order to obtain the required film formation that best meets the device specifications. Between the several microscopy techniques currently employed, Amplitude Modulation Atomic Force Microscopy (AM-AFM) is a powerful, flexible and economic tool that enable us to study the surface and the heterostructure morphology. Like roughness analyses [3], grain structure [4], cross section heterostructure features, chemical sensitivity and materials properties through the phase contrast operation mode [5]-[8]. Certainly, by AM-AFM, it is possible to exploit both signals: the topography and the phase channels. Exploring the phase signal capabilities is of particular interest because of its sensitivity to materials properties [9] [10], which can lead to interesting and useful analysis of cross section semiconductor heterostructures. This transversal AM-AFM analysis, enable us to study not only the surface structure, but also the inner features of any multi-composed assemblage. For all this, in this work, present the results of the characterization of the GaAs/In bilayer grown by Magnetron RF Sputtering, to study the influence of the deposition time of the Indium (In) layer on morphological and optical properties of the bilayer. The AM-AFM characterizations of the three samples prepared were implemented in terms of surface morphology, cross-section imaging composition. Surface morphology was described in terms of the following statistical quantities: Maximum Height value of the grains and Root Mean Square (RMS) roughness. Grain analysis, by using the watershed algorithm [4], was implemented to obtain the following quantities: Grain Population Density, Mean Grain Size and Total Grain Volume. The inner heterostructure features was studied in terms of cross section imaging, combining the topography features with the phase shifts signal, to observe the film thickness and the topography and composition effects on the phase channel.

The manuscript is developed as following. We will start with the experimental setup section, where a description of the surface preparation, the AM-AFM implementation is given. We will continue with the results portion, where AFM images of the three specimens are showed. After that, the discussion part will be presented, where the grain and cross sectional analysis is made, and finally the conclusion part.

\section{Experimental Setup}

\subsection{Sample Preparation and Fabrication}

The In/GaAs bilayers where grown on a (100) silicon substrate by Radio Frequency Magnetron Sputtering in an Argon (Ar) atmosphere. High purity (95.95\%) GaAs (100) and In targets where used. Before deposition the Si (100) surfaces were firstly degreased with acetone and methanol. Secondly, they were cleaned with hydrofluoric acid at $2 \%$, rinsed with deionized water, and dried by flowing nitrogen. Thirdly, the substrate was introduced into the sputtering chamber to start the vacuum process. When the base pressure of $1.2 \times 10^{-6}$ Torr was reached, the substrate temperature was raised up $650^{\circ} \mathrm{C}$ to remove the oxide on surface. The substrate temperature was lowered and stabilized at $580^{\circ} \mathrm{C}$, and high purity Argon gas was introduced into the chamber until a working pressure of $1.2 \times 10^{-3}$ Torr, and the plasma is turning on. Next, the GaAs target source was powered on in order to deposit a GaAs buffer layer during 30 min, after then the GaAs shutter was closed. Subsequently, the substrate temperature was lowered to $300^{\circ} \mathrm{C}$ and the Indium (In) source target was powered on, in order to deposit Indium during $t_{d}=10$ minutes (sample M1), 15 minutes (sample M2) and 20 minutes (sample M3), respectively. This process is then repeated once again in order to deposit two In/GaAs films. Finally, the structure was capped with a GaAs layer ( $\left.\mathrm{t}_{\mathrm{d}}=20 \mathrm{~min}\right)$.

\subsection{AFM Equipment Setup Description}

The AM-AFM characterization where performed with the N8 NEOS SENTERRA system from Bruker, working at ambient conditions. This equipment combines an optics SENTERRA Raman spectrometer with the Nano's N8 NEOS Atomic Force Microscope, to allow the morphological, structural and chemical analysis of the same sample area. It is mounted on a very heavy table and everything is enclosed in a Faraday Box. The cantilever beam deflection sensor is based in a fiberoptical interferometric detection system. The cantilevers used were Point Probe Plus Non-Contact Long cantilever Reflex coated (PPP-NCLR) from Nanosensors, with resonance frequencies in vacuum of $190 \mathrm{Khz}$, and force constants around $48 \mathrm{~N} / \mathrm{m}$. The AFM machinery was set to work in AM-AFM contrast phase mode, with free oscillation amplitudes of $195 \mathrm{~nm}$, set points of 50\%, 65\% and 80\% and oscillation frequencies around $169 \mathrm{Khz}$. All the AFM images presented here shown the forward scanning 
direction. Free oscillation phase was set to $90^{\circ}$, in a range going from $0^{\circ}$ to $180^{\circ}$ or from $0^{\circ}$ to $-180^{\circ}$. The software's programs used to treat the AFM images were Gwyddion [11], WSxM of "Nanotec Electronica" [12] and Nano Scope Analysis [13] (the sample orientation with respect to the AFM cantilever is shown in Appendix).

\section{Analysis and Results}

Figure 1 shows the optical images obtained with a 100 $\times$ lent (upper part) and the corresponding $40 \times 40 \mu \mathrm{m}^{2}$ AM-AFM topography images (lower part), for each of the three samples: In Figure 1(a), Figure 1(d) and Figure $1(\mathrm{~g})$, we have the 5 minutes double cycle of Indium deposition, corresponding here to the sample M1. The Figure 1(b), Figure 1(e) and Figure 1(h) displays the 10 minutes double cycle of indium deposition, sample M2. And Figure 1(c), Figure 1(f) and Figure 1(i) show the 15 minutes double cycle of Indium deposition, sample M3. For each sample, the optical images presented at the upper part, Figures 1(a)-(c), are marked with a blue square, which is the zone where the AFM imaging took place, Figures 1 (d)-(f).

For these large scale AFM images, the corresponding statistical quantities were the following. For the sample M1 it was found a Maximum Height of $2.36 \mu \mathrm{m}$ and the RMS roughness was $0.28 \mu \mathrm{m}$. For the sample M2, we found a Maximum Height of $3.18 \mu \mathrm{m}$ and the RMS roughness was $0.38 \mu \mathrm{m}$. Finally, for the sample M3, we found a Maximum Height of $7.72 \mu \mathrm{m}$ and the RMS roughness was $0.97 \mu \mathrm{m}$.

In order to obtain the grain discretization a $10 \times 10 \mu \mathrm{m}^{2}$ zoom was obtained in this same area for each sample. The corresponding AFM topographic images are following shown in Figure 2.

From the Figure 2(a), sample M1, where the Indium deposition time was two cycles of five minutes, we obtained the following statistical quantities: $1.79 \mu \mathrm{m}$ of Maximum Height and $0.24 \mu \mathrm{m}$ of RMS roughness. The grain statistics obtained from Figure 2(a), and discretized in Figure 2(d), were: number of grains 1120, grain

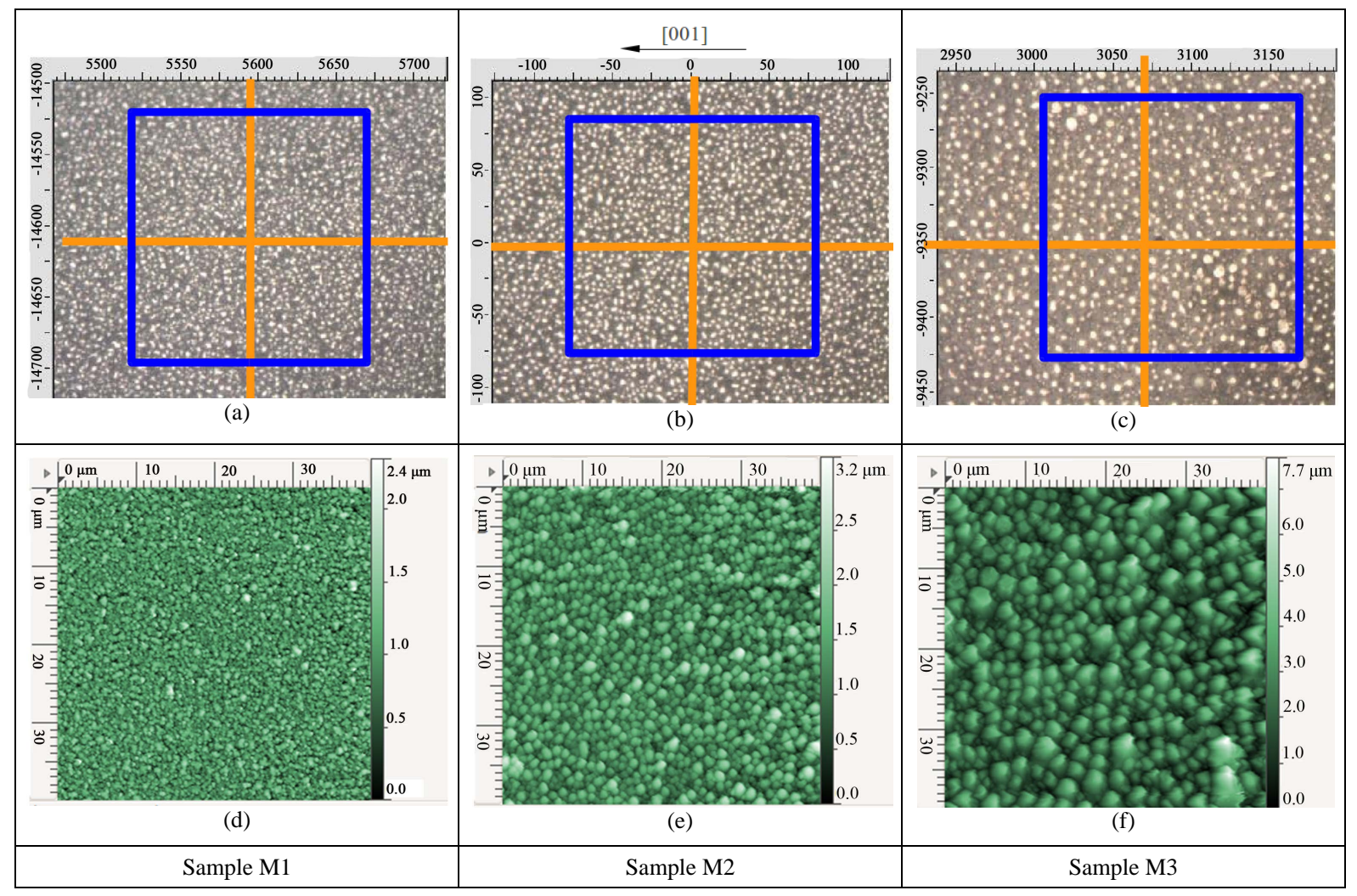

Figure 1. 100× optical image (upper part), large scale $40 \times 40 \mu \mathrm{m}^{2}$ AM-AFM topography images (lower part). Column 1 ((a), (d)), column 2 ((b), (e)) and column 3 ((c), (f)), show the corresponding results for the sample M1, the sample M2 and the sample M3 respectively. The respective AFM parameters for the (d) were: set point (SP) $=50 \%$, free amplitude (FA) = $195 \mathrm{~nm}$, oscillation frequency $(\mathrm{OF})=169.12 \mathrm{Khz}$ and scanning speed $(\mathrm{SS})=0.5 \mathrm{l} / \mathrm{s}$. For the (e), the imaging parameters were: $\mathrm{SP}=50 \%, \mathrm{FA}=195 \mathrm{~nm}, \mathrm{OF}=169.32 \mathrm{Khz}$ and SS = 0.7 l/s. And for (f): $\mathrm{SP}=50 \%, \mathrm{FA}=195 \mathrm{~nm}, \mathrm{OF}=169.37 \mathrm{Khz}$ and SS $=0.5 \mathrm{l} / \mathrm{s}$. The corresponding [001] directions are pointing towards the left for all the specimens. 


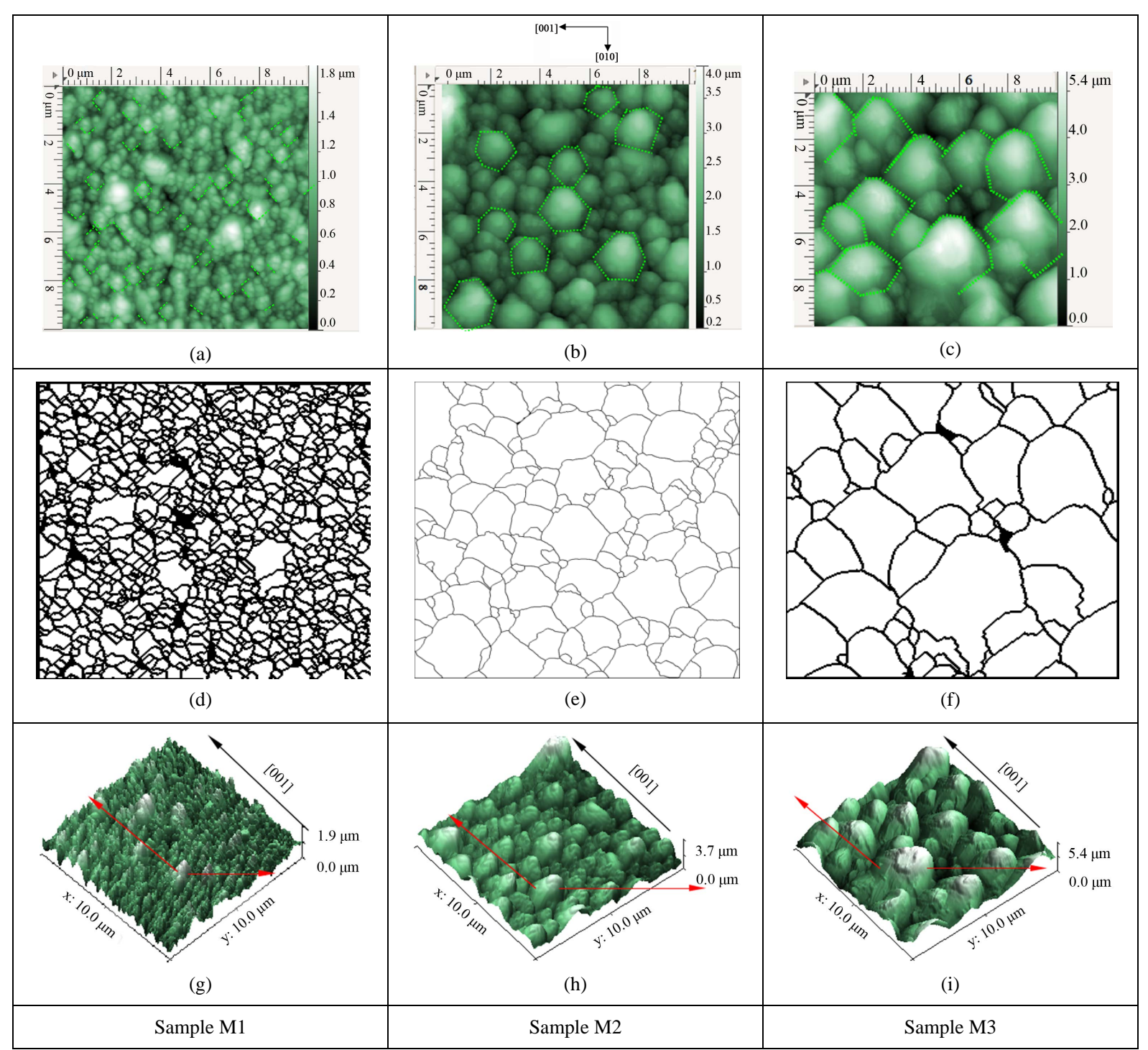

Figure 2. A $10 \times 10 \mu \mathrm{m}^{2}$ AM-AFM topography images for (a) sample M1, (b) sample M2, and (c) sample M3. Their corresponding watershed grain discretization mask for each image is shown in the middle part ((d), (e), (f)). The AFM parameters were: (a) SP = 50\%, FA = $195 \mathrm{~nm}, \mathrm{OF}=169.35 \mathrm{Khz}, \mathrm{SS}=0.5 \mathrm{l} / \mathrm{s}$; (b), SP = 65\%, FA = $195 \mathrm{~nm}$, OF = $169.27 \mathrm{Khz}$ and SS = $0.6 \mathrm{l} / \mathrm{s}$; (c) $\mathrm{SP}=50 \%, \mathrm{FA}=195 \mathrm{~nm}, \mathrm{OF}=169.37 \mathrm{Khz}$ and SS = $0.5 \mathrm{l} / \mathrm{s}$; (d)-(f) We show the grain discretization for the samples M1, M2 and M3 respectively, obtained from the corresponding images (a), (b) and (c). The 3D-representations images of (a), (b) and (c) are shown in (g), (h) and (i) respectively. The corresponding [001] directions are pointing towards the left for all the samples.

population density $11.2 \mu \mathrm{m}^{-2}$, Mean Grain Size $0.183 \mu \mathrm{m}$ and Total Grain Volume $48 \mu^{3}$. For the sample M2 the Indium deposition time was two cycles of ten minutes, and from Figure 2(b) we obtained the following statistical analysis: Maximum Height, $3.77 \mu \mathrm{m}$ and RMS roughness, $0.51 \mu \mathrm{m}$. The grain statistics obtained from Figure 2(b) and discretized in Figure 2(e) were: Number of grains 171, Grain Population Density $1.71 \mu^{-2}$, Mean Grain Size $0.63 \mu \mathrm{m}$, Total Grain Volume $137 \mu^{3}$. And for the sample M3, where the Indium deposition time was two cycles of fifteen, Figure 2(c), the statistical analysis were: Maximum Height, $5.44 \mu \mathrm{m}$ and RMS roughness, $1.01 \mu \mathrm{m}$. The grain statistics obtained from Figure 2(c) and discretized in 2(f) were: Number of grains 73, Grain Population Density $0.73 \mu \mathrm{m}^{-2}$, Mean Grain Size $0.82 \mu \mathrm{m}$ and Total Grain Volume, $227 \mu^{3}$.

An interesting observation presented by the AFM topography images, was the presence of linear features. The Figure 2(a) of the sample M1, presented several linear features arranged in a perpendicular way and giving rise to a repetitive pattern of corners alike forms, which apex is pointing to the [010] direction, here indicated by the 
green dotted lines. For the sample M2, this corner alike shapes evolved to the hexagonal alike shapes indicated by the green dotted lines of Figure 2(b). And for the sample M3, hexagonal, rectangular and polygonal alike shapes were found. Again there are indicated with the green dotted lines on Figure 2(c). Either the perpendicular features as well as the polygonal alike shapes, seems to be randomly localized. Note that the localization of these shapes is easily obtained from the grain discretized mask for the samples M2 and M3 but more difficult for the one representing the sample M1. Another interesting observation was obtained from the 3D representations, shown in Figures 2(g)-(i), for the samples M1, M2 and M3 respectively. Here, it is possible to note that the grain structures were presented in all the specimens; however a larger tilted planes with certain acute angles. This is more clearly appreciated for the samples M2 and M3, as following described.

The topography AFM images presented in Figure 3, shows a zoomed area of $5 \times 5 \mu \mathrm{m}^{2}$ of the three samples. In this part of the specimens one of the larger grains found for each sample, was selected to be measured by a height profile line. These measurements showed these tilted planes (see Figure 3).

Figure 3 shows now a series of height measurements for each topography image corresponding to each sample. In this area, the highest height was $1.84 \mu \mathrm{m}, 3.49 \mu \mathrm{m}$ and $5.4 \mu \mathrm{m}$, for the samples M1, M2 and M3, respectively. The image shown in Figure 3(a) is marked with black dotted lines some of the linear features found on the specimen. In this case, the highest height found is the product of an island, an agglomeration of grains. As the indium quantity increased, these agglomerations leaves to the formation of bigger grains giving rise to the bigger grains presented in Figure 3(b) and Figure 2(c), for the samples M2 and M3 respectively. The profile shown in every AFM image is presented with their corresponding Height vs Distance Graph (lower part). The images of Figure 3(b) and Figure 3(c), upper part, presented the tilted plane more clearly upon subsequent Indium deposition, here marked by the black dotted line triangle. The height profile measured on the biggest grains presented by the AFM images, shown that these tilted planes presented acute angles $\theta$, from the [011] direction, having values of about $53^{\circ}, 58.5^{\circ}$ and $61^{\circ}$, for the samples M1, M2 and M3 respectively. Furthermore, towards this same [011] direction, the top of the clusters seems to be smoothed upon increments of the Indium quantity. While the sample M1 presented a sharper structure on the top, the sample M2 presented a less sharp

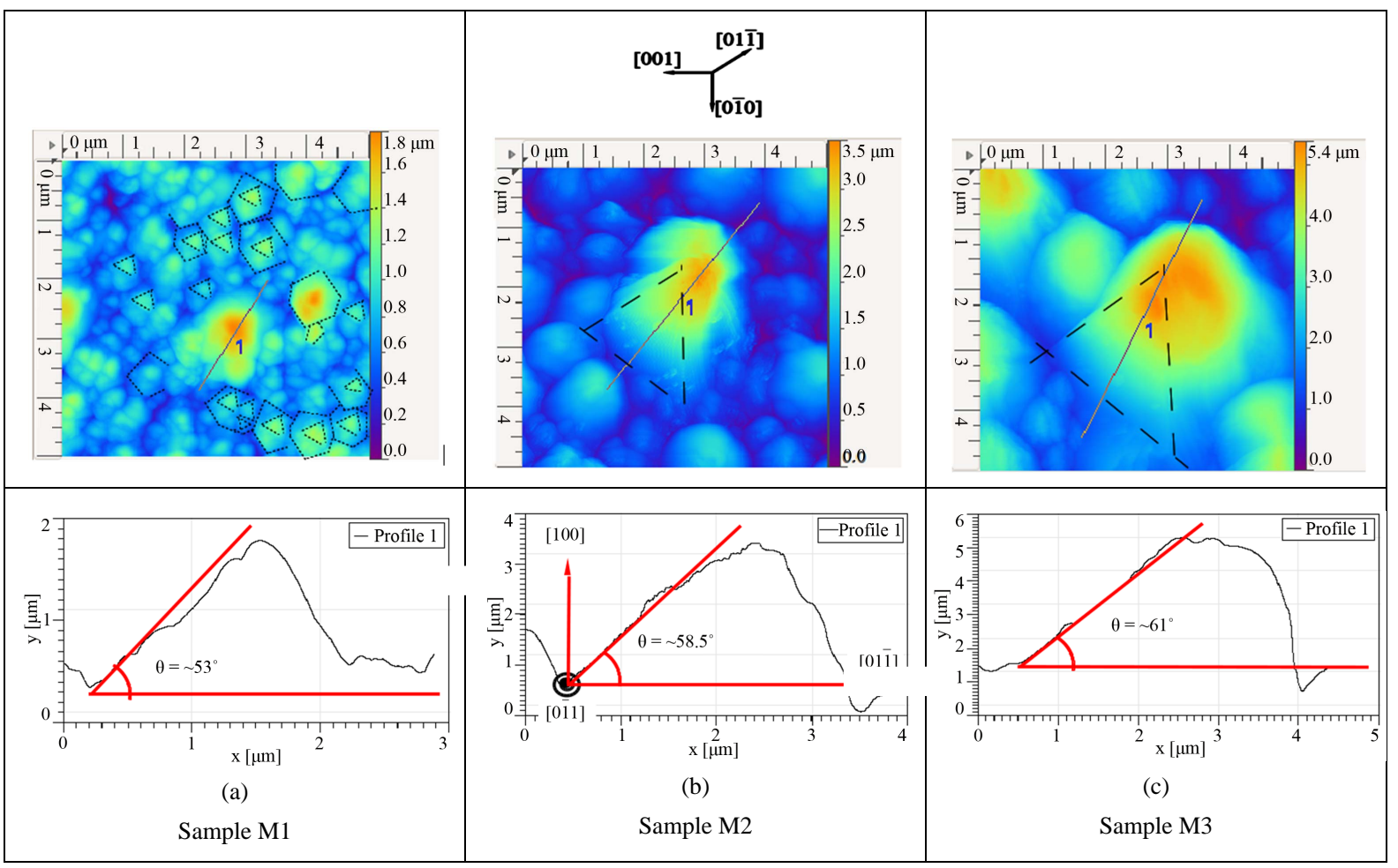

Figure 3. $5 \times 5 \mu^{2}$ topography AFM images in warp-spectral color representation. For each sample, the images at the upper part, contains the line profile, marked with number 1 . In the lower part we see the height profile for each image. The corresponding [001] direction is pointing to the left side, the [010] goes toward the bottom and the [011] is pointing to the diagonal top-right part of the image. 
shape, with a small planar section. On the other hand, the sample M3 seemed to have small terraces separated by certain heights.

In the following, the cross section experiments are presented.

\section{Cross Section Imaging: Topography and Phase Micrographs}

The following images shows the cross section morphology of the samples, see Figure 4. On top, we can see the optical images with the 100× lent. Below it is shown the corresponding topography AM-AFM image of the marked zone by the green rectangle area in each of the optical images.

The optical images of Figure 4, shows the position of the In/GaAs/In/GaAs film with the red arrow, pointing toward the right side of the image, the [100] direction. The Si substrate is presented at the left side and the black color to the right is the free space after the specimen. Note that the $3 \times 10 \mu \mathrm{m}^{2}$ AM-AFM topographic images, preserves the same orientation than that of the optical image. These AFM images with their height profiles are following compared to their corresponding phase images, presented with their corresponding Phase vs Distance Graphs, in Figure 5. A Code-V color representation for the topography and phase AM-AFM images was used. The presence of the In/GaAs/In/GaAs film is appreciated more clearly. The topographic channels are presented in the upper part, Figures 5(a)-(c) for the samples 1, 2 and 3, respectively. Here, the silicon substrate is exhibited with orange, hard-orange or red colors. The GaAs-In film is presented with yellow and/or with green color. Each image has a height profile line, which is shown below. In this particular locality, these height profiles show a film thickness of about $2.16 \mu \mathrm{m}$ for the sample M1, $3.85 \mu \mathrm{m}$ for the sample M2, and $6.15 \mu \mathrm{m}$ for the sample M3. Note that these values are quite close to those observed by the height profiles of the big grains presented in Figure 3. Here the criteria followed to obtain this particular distance, was by selecting the first abrupt change in the phase channel that was followed by a different phase behavior. For the sample M1, Figure 5(a), the height profile starts with a linear tendency from the left (light orange color). Then the line profile presented a small increment until a maximal height value of $6 \mu \mathrm{m}$ (orange color), that decreased until a height of $5.29 \mu \mathrm{m}$ at about $7.52 \mu \mathrm{m}$ of distance. This is what we considered to be the part of the Si substrate. After this the height value started to decrease to about $4.5 \mu \mathrm{m}$ of height and remained there for a while (corresponding to the yellow contrast in Figure 5(a)). Then the height decreased linearly (green contrast) until a value close to $0.73 \mu \mathrm{m}$ of height

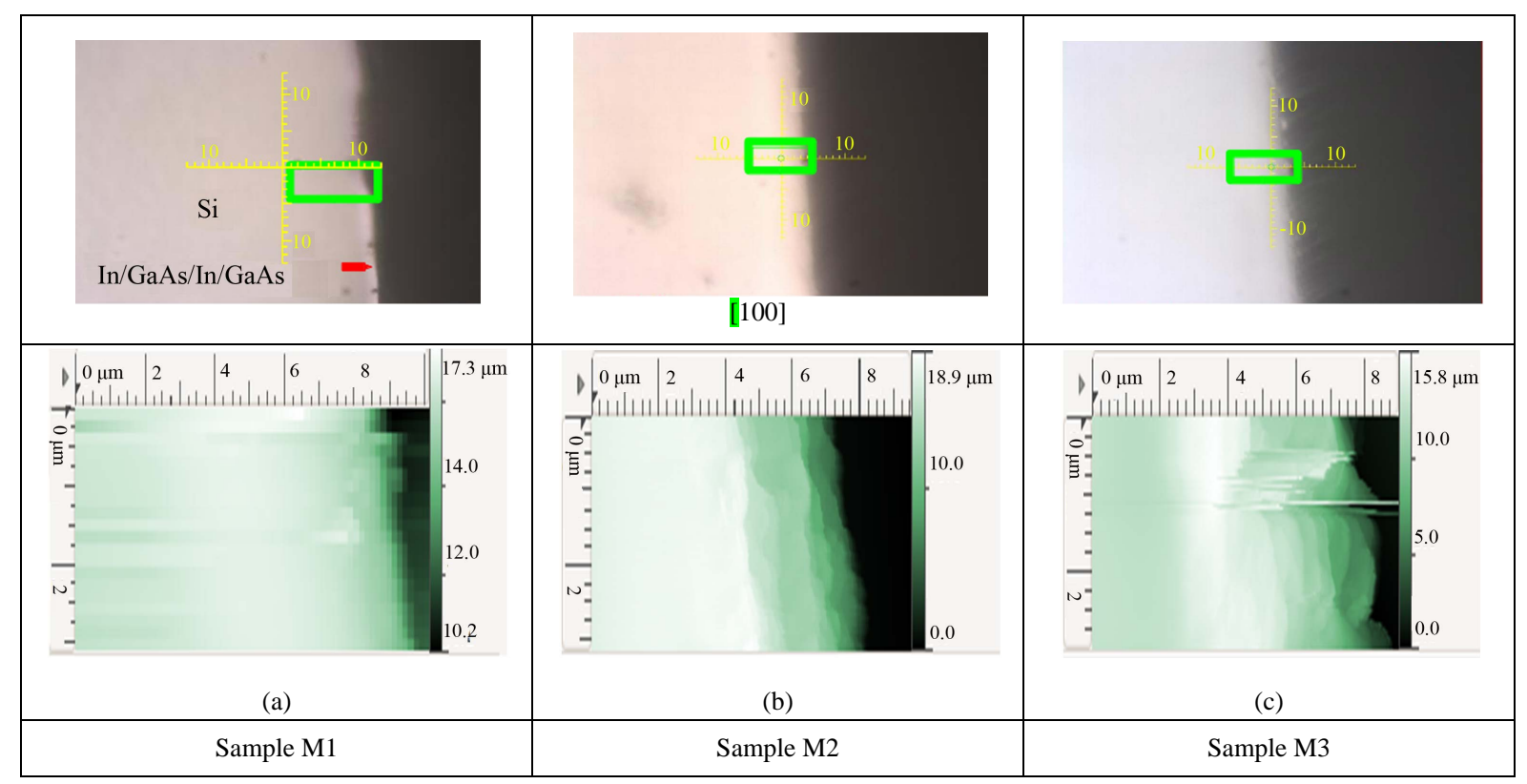

Figure 4. Optical images and the corresponding $3 \times 10 \mu \mathrm{m}^{2}$ AM-AFM topography images of the cross section, for each of the sample. The $100 \times$ optical images are presented at the upper part, while the AM-AFM topography images are shown below. In (a) SP $=50 \%$, OF $=169.12 \mathrm{Khz}$ and SS $=0.7 \mathrm{l} / \mathrm{s}$; In (b) SP $=80 \%$, OF = $163.4 \mathrm{Khz}$ and SS = $0.2 \mathrm{l} / \mathrm{s}$; In (c) SP = $50 \%$, OF $=163.27 \mathrm{Khz}$ and SS $=0.2 \mathrm{l} / \mathrm{s}$. The corresponding [100] directions for all the samples are indicated on top of the column (b). 


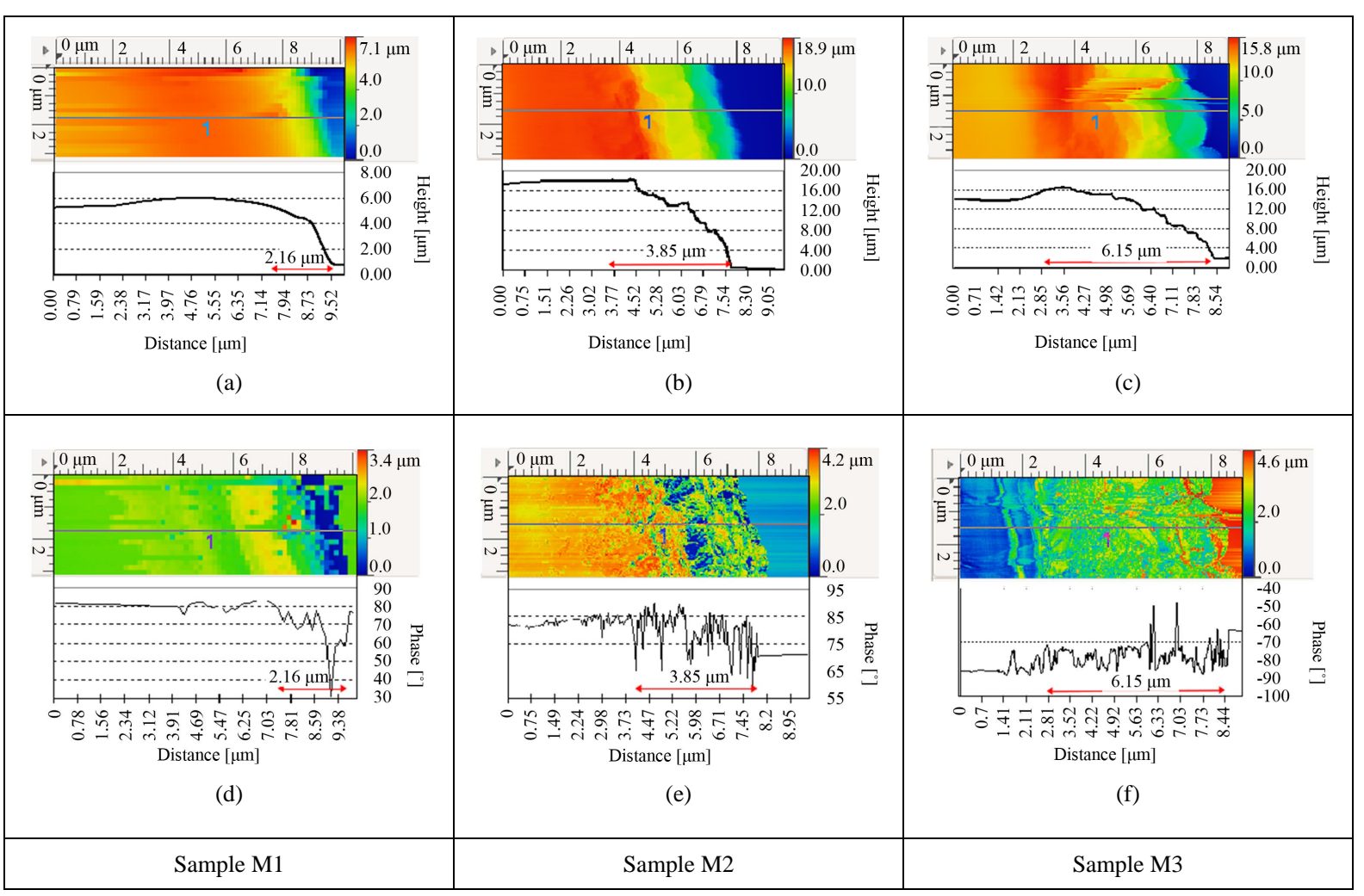

Figure 5. $3 \times 10 \mu \mathrm{m}^{2}$ AM-AFM topography and phase images of the cross section, for each of the samples, in Code-V color representations. They are presented with their corresponding height profiles and their phase shifts vs distance curves.

at $9.68 \mu \mathrm{m}$ of distance. Finally a constant value remained at this point (the blue contrast) showing that the tip is not any more in contact with the cross section of the specimen. Regarding the phase signal, Figure 5(d), it started with a linear tendency at about $80^{\circ}$ (green contrast) starting from the left part of the image. Then the presence of some perturbations to $75^{\circ}$ and $83^{\circ}$ until a distance of $6.25 \mu \mathrm{m}$, followed by a small lobe to $85^{\circ}$, appeared (yellow color). After this, the phase signal decreased to $76^{\circ}$ at a distance of $7.5 \mu \mathrm{m}$, (green color), and then increments and decrements to $77^{\circ}$ and $68^{\circ}$ are continued (green, yellow, blue colors) until a clear fallen of the phase to $31^{\circ}$ at $9.1 \mu \mathrm{m}$ of distance (blue color). From here the phase went up to $62^{\circ}$ and then to $77^{\circ}$ at 9.68 $\mu \mathrm{m}$ of distance, where it remained until the end of the profile part, right side of the image (green color) corresponding to the free oscillation of the cantilever.

For the sample M2, Figure 5(b), a similar feature is presented in the height profile measurement, but not with the same increment rate as it was presented in sample 1 . Starting from the left side, a more straight linear tendency at about $17.82 \mu \mathrm{m}$ is presented until a distance of about $4.06 \mu \mathrm{m}$ (all the hard-orange/red color). This part is what we attributed to be the Si substrate. A small lobe is presented just before the height started to decrease. Then the profile is decreased (yellow contrast) until the profile goes to a value of about $13 \mu \mathrm{m}$ of height and remained there for a while (green contrast). Then the height is decreased until a $0.44 \mu \mathrm{m}$ of height is reached, at $7.91 \mu \mathrm{m}$ of distance, where the signal remained until the end of the profile at the right part of the image (all the blue color). Here is where the cantilever is in the free space position. The phase presented values near $81^{\circ}$, corresponding to the orange and red contrasts, left side of Figure 5(e), and then the phase increased gradually until a close value of $86^{\circ}$, around a distance of $2.98 \mu \mathrm{m}$. Here it is presented a series of jumps near $85^{\circ}$ until a fast fallen to $64.95^{\circ}$ at $4.06 \mu \mathrm{m}$ of distance. After this, increments to values close to $85^{\circ}$ with intermittent jumps to $90^{\circ}$ were presented (red, blue, green and orange zone) until a distance of $5.6 \mu \mathrm{m}$, where a noticeable decrease in the phase is observed. Here the phase presented different fallen and jumps to $75^{\circ}$ and up to $85^{\circ}$ (corresponding to the blue, green and yellow colors) and lasted until a distance of $7 \mu \mathrm{m}$. Then the phase presented jumps from $65^{\circ}$ to $85^{\circ}$ and $80^{\circ}$. These phase oscillations were attenuated until a close value to $70.23^{\circ}$ at $7.9 \mu \mathrm{m}$ of distance, where the attenuated phase kept a constant value (blue contrast), presented at the right part of the image. This is 
the part where the cantilever is not any more interacting with the specimen cross section.

Finally, for the sample 3, starting from the left part of the image, the height profile presented a constant height value, at about $13.8 \mu \mathrm{m}$, which lasted to a distance of $2.13 \mu \mathrm{m}$. Then an increment to about $14.68 \mu \mathrm{m}$ of height started at about $2.46 \mu \mathrm{m}$ of distance (orange and red color). This is what we considered to be the Si substrate. The profile remained for a while around $16 \mu \mathrm{m}$ and then decreased with a logarithmic alike behavior (yellow and green colors) for all over a distance of $5.49 \mu \mathrm{m}$ and until a value near $1.75 \mu \mathrm{m}$ of height, at $8.52 \mu \mathrm{m}$ of distance. From here a constant value remained all over the right side of the image (blue contrast). Regarding the phase signal of the sample 3 , the image started to the left part (blue contrast), corresponding to values near $-85^{\circ}$, Figure 5(f). At $1.41 \mu \mathrm{m}$ of distance, a pick to $-75^{\circ}$ was observed, then returning to $-85^{\circ}$, followed by a decrement to around $-90^{\circ}$ at $2.11 \mu \mathrm{m}$ of distance. Two picks are then presented, one to $-7.7^{\circ}$ at $2.46 \mu \mathrm{m}$ and the other to $-71^{\circ}$ at $2.7 \mu \mathrm{m}$ (green and blue colors). After that, values around $-75^{\circ}$ with intermittent fallen to $-80^{\circ}$ were presented until a distance of about $6 \mu \mathrm{m}$ (green, blue, yellow colors). Then a sharp pick to $-45^{\circ}$ with a subsequent fallen to $-80^{\circ}$ followed by another pick to $-50^{\circ}$ and a consecutive fallen to $-75^{\circ}$ were presented. The phase went down to $-85^{\circ}$ with jumps to $-70^{\circ}$ and fallen phases to $-80^{\circ}$ (green, yellow, blue, hard-orange colors) until a constant value of around $-63.61^{\circ}$ at $8.61 \mu \mathrm{m}$ of distance. At this phase value the signal was maintained until the end of the profile, at the right part of the image (hard-orange color).

To conclude with this section, the phase signal presented a different value in the GaAs/In/GaAs/In/GaAs film compared with the silicon substrate. It is important to note that the change in phase is presented in conjunction with a change in topography; however, even though the change in topography decreased with a logarithm alike behavior, the phase shift did not. Then after the existence of the cross section, were the tip was oscillating in the free space, the phase shift was not $90^{\circ}$ as it was originally when far away from the surface.

Regarding the AFM study, from the watershed algorithm of the Gwyddion software, we could obtain the discretization of the grains and the corresponding mask, containing the grain boundaries for each of the three samples studied, Figures 2(d)-(f). From these masks, and with the use of the Maximum Height statistical quantities obtained from each of the AFM images, we could observe that the grain lateral dimensions and heights, as well as the film averaged thickness, were increased upon larger Indium deposition times. This observation is consistent with the Mean Grain Size and total gran volume quantities extracted from the grain statistics see Table 1. This suggests that there exists a growing tendency produced by the number of Indium layers deposited and the grain size. The AFM images presented in Figure 2, showed the following behavior. Figure 2(a) presented a variety of very small grains that in certain zones they formed agglomerations (islands), that exhibited higher heights and greater lateral dimensions. These agglomerations were not homogeneous around the $10 \times 10 \mu \mathrm{m}^{2}$ surface. Instead, they apparently seem to be randomly arranged. By subsequent Indium deposition, theses agglomerations collapsed and formed bigger grains, leaving the new smaller grains to accumulate around the bigger ones, see Figure 2(b). These bigger grains started to present a large tilted plane towards the [011] direction, with acute angles that increased from $53^{\circ}, 58.5^{\circ}$ and $61^{\circ}$ while the Indium quantity increased. Also the plane in front of this tilted plane tended to form right angles from the [011] horizontal line and the top of the grains became flatter. This suggest a pure three dimensional growth with a high tendency of expansion towards the [100] direction. This three dimensional growth comes from the lattice parameters mismatches presented by the substrates and the alloys.

Regarding the statistical quantities and grain statistics, we obtained the Table 1 and relations.

The increments of the In deposition time, starting from a total of 20 minutes for the sample M1, was scaled

Table 1. Comparative table of the statistical quantities and grain statistics between samples.

\begin{tabular}{|c|c|c|c|c|c|c|c|}
\hline \multirow[b]{2}{*}{ Sample } & \multirow[b]{2}{*}{$\begin{array}{l}\text { Total time of GaAs } \\
\text { deposition (min) }\end{array}$} & \multirow[b]{2}{*}{$\begin{array}{l}\text { Total time of In } \\
\text { deposition (min) }\end{array}$} & \multicolumn{2}{|c|}{ Statistical quantities } & \multicolumn{3}{|c|}{ Grain statistics } \\
\hline & & & $\begin{array}{c}\text { Maximum } \\
\text { Height } \\
(\mu \mathrm{m})\end{array}$ & $\begin{array}{c}\text { RMS } \\
\text { roughness } \\
(\mu \mathrm{m})\end{array}$ & $\begin{array}{c}\text { Grain Population } \\
\text { Density } \\
\left(\mu \mathrm{m}^{-2}\right)\end{array}$ & $\begin{array}{c}\text { Mean Grain } \\
\text { Size } \\
(\mu \mathrm{m})\end{array}$ & $\begin{array}{l}\text { Total Grain } \\
\text { Volume } \\
\left.\left(\mu^{3}\right)^{3}\right)\end{array}$ \\
\hline M1 & 60 & 20 & 1.79 & 0.24 & 11.2 & 1.18 & 48 \\
\hline M2 & 60 & 30 & 3.78 & 0.51 & 1.71 & 0.63 & 137 \\
\hline M3 & 60 & 40 & 5.44 & 1.01 & 0.73 & 0.82 & 227 \\
\hline
\end{tabular}


from this value by a factor of 2, corresponding to the 30 minutes for the sample M2, and by a factor of 3, corresponding to the 40 minutes for the sample M3. These scaled factors are similar to the increasing proportions found in the Maximum Height. On the other hand, the RMS roughness values measurements, presented the following features. A scale factor of 2.16 was found from the 0.237 of the sample 1 , to a value of $0.512 \mu \mathrm{m}$ of the sample 2, and a factor of $4.27 \mu \mathrm{m}$ to $1.01 \mu \mathrm{m}$ of the sample 3. The Grain Population Density was found to be scaled from 11.2 of the sample 1 to 1.71 of the sample 2, by a factor of 0.15 , and to 0.73 of the sample 3 , by a scale factor of 0.07 . The Mean Grain Size was found to be scaled from 0.18 of the sample 1 by scale factors of 3.44 and 4.48 to $0.63 \mu \mathrm{m}$ and $0.820 \mu \mathrm{m}$ of the sample 2 and the sample 3, respectively. Finally, the Total Grain Volume was found to be scaled by 2.85 and 4.73 from $48 \mu \mathrm{m}^{3}$ of the sample 1 to $132 \mu \mathrm{m}^{3}$ of the sample 2 and $227 \mu \mathrm{m}^{3}$ of the sample 3 . The height related features were found to be scaled by 2 and 3 scale factors, as well as the symmetrical factors found by the RMS roughness and Grain Population Density. However, the Grain Population Density as well as the Total Grain Volume presented asymmetrical scaled factors. From the sample 1, the Grain Population Density presented a value of $11.2 \mu^{-2}$ and by increasing the double In quantity for the sample 2 fabrication, the Grain Population Density decreased to $1.71 \mu \mathrm{m}^{-2}$. Meaning that around this increment of Indium quantity a large number of clusters were collapsed into Islands and then the islands into grains. This was not the case when passing to the fifteen minutes double cycle for the fabrication of the sample M3, where the Grain Population Density was 0.73

Regarding the phases analysis, for the moment only qualitative information can be given, see Figure 6 .

As shown in Figure 1, for the sample 1, this particular profile line scan speed was made at $0.7 \mathrm{l} / \mathrm{s}$, which is faster than those speeds of $0.2 \mathrm{l} / \mathrm{s}$ used for imaging the sample 2 and sample 3 . However, while the topography signal started to decrease in a logarithmic form, the phase signal presented a linear tendency composed of jumps and falls. The topography effect observed on the Phase signal was, when the oscillating tip found an abrupt fall in the topography, a fallen for the sample 1 and 2 and a jump for the sample 3 . This falls and jumps are indicated by the dotted green lines in the Figures 6(a)-(c). The overall behavior of the phase signal did not show any logarithmic or exponential tendency. For example in the sample 2, when the topography signal decreased after the small lobe, at around $4.5 \mu \mathrm{m}$ of distance, the phase fallen form a while. Then it seems to be oscillating until a more stable signal was obtained. When the topography went down to $13 \mu \mathrm{m}$ of height, the phase suffered also a fallen. Then it came back to around $70^{\circ}$ and even thought the topography profile, started to decrease the phase signal presented values around $70^{\circ}$, with intermittent falls and jumps. No exponential or logarithmic behavior was exhibited by the phase. This behavior is still more presented for the sample 3, Figure 6(c). Here, the topography profile clearly decreased logarithmically; while the phase seemed to presents a repetitive value around $-75^{\circ}$. Again when the topography signal presented a suddenly fall, at around $6.11 \mu \mathrm{m}$, the phase presented a big pick to around $-50^{\circ}$, then came back to values close the $-75^{\circ}$. Another abrupt fall in the topography signal at 6.90 $\mu \mathrm{m}$ was presented, and again, the phase presented a big jump to around $-45^{\circ}$, and then returned to the values

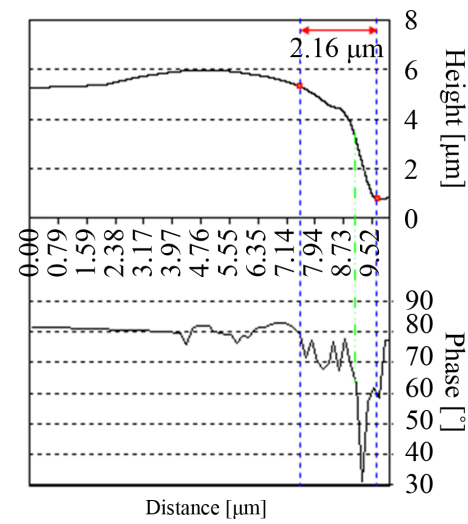

(a)

Sample M1

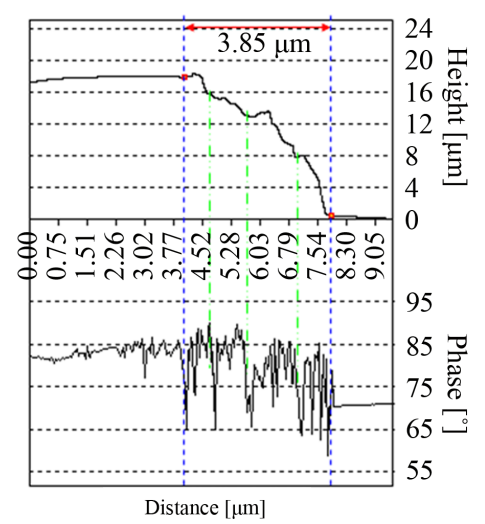

(b)

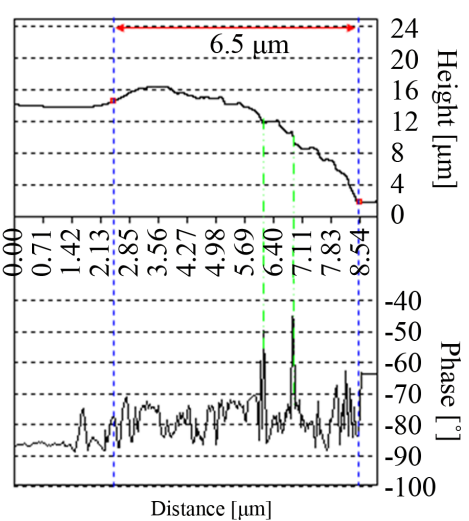

(c)

Figure 6. Qualitative comparative analysis of the topographic and phase signals. In (a) the scanning speed (SS) of the AFM image for the sample M1, was $0.7 \mathrm{l} / \mathrm{s}$; In (b) SS = $0.2 \mathrm{l} / \mathrm{s}$ for the sample M2 and in (c) SS = $0.2 \mathrm{l} / \mathrm{s}$, for the sample M3. 
between $-80^{\circ}$ and $-70^{\circ}$.

Finally, regarding the corners shapes found in the sample 1, the hexagonal shapes found in the sample 2 and sample 3 as well as the grain three dimensional structures, the growth process of In-GaAs on top of clean Si (100) substrates probably leads to the formation structures with some order that runs in certain crystallographic orientations. A subsequent analysis by electron microscopy can give us new insides about these observations as well as a detailed study of the initial growth of GaAs on $\mathrm{Si}$.

\section{Conclusions}

Three different samples containing In/GaAs films on Si (100) substrates with different Indium quantity were successfully fabricated by RF Magnetron Sputtering. The samples were successfully characterized by AMAFM.

The AFM was implemented in order to obtain the morphology of the surface and of the inner structure features for each of the samples. For a total time of 20 minutes of In deposition, small grains were formed. Corner alike shapes were found, and some agglomerations of grains were presented. This initial formation presented a certain growth tendency towards the [111] direction and a tilted plane towards the [011] direction could be identified. For a total of 40 minutes, this agglomerations formed bigger grains, and for a total of 30 minutes of In deposition these grains presented larger tilted planes and a flatter structure on top.

Thanks to the statistical quantities obtained, we could observe that the samples reported Maximum Heights of 1.79, 3.77 and 5.44 $\mu \mathrm{m}$, presenting RMS roughness of 0.237, 0.512 and $1.01 \mu \mathrm{m}$ for the sample M1, M2 and M3, respectively. Thanks to the implementation of the watershed algorithm, these grains could be discretized. From where the grain population densities of $11.2 \mu \mathrm{m}^{-2}, 1.71 \mu \mathrm{m}^{-2}$ and $0.73 \mu \mathrm{m}^{-2}$ where obtained. The Mean Grain Size, varied from $183 \mathrm{~nm}$ to $630 \mathrm{~nm}$ and to $820 \mathrm{~nm}$, and the Total Grain Volume varied from $48 \mu \mathrm{m}^{-3}$ to 137 $\mu \mathrm{m}^{-3}$, and then to $227 \mu \mathrm{m}^{-3}$. These grains formed some shapes that could be identified. The corners shapes found in the sample 1, the hexagonal shapes found in the sample 2 and polygons found in the sample 3 as well as the grain three dimensional structures, leads to the supposition that the growth process of In-GaAs on top of clean Si (100) substrates probably give rise to the formation of structures with some order that runs in certain crystallographic orientations. A subsequent analysis by electron microscopy can give us new insides about these observations as well as a detailed study of the initial growth of GaAs on Si.

Finally, the qualitative analysis observed here, the cross section phase imaging presented different phases shift for each sample, comparing the Si (100) and the In/GaAs film. The more notable result was that while the topography profile decreased logarithmically, the tendency of the phase signal did not present this behavior. The sudden falls on the topography signal, produced a sharp pick in the phase signal. As an idea, it is clear that Silicon, Gallium Arsenide and Indium Arsenide have different mechanical properties, like different harness values. It would be very interesting to try to relay any mechanical properties of the alloys and substrates compositions with the phase signal evolution. But for the moment we are not able to relate anmechanical properties with the phase shifts.

\section{Acknowledgements}

We are very much appreciated with the "Instituto de Ciencia y tecnologíadel Distrito Federal, (IcyTDF)” now the "Secretaría de Ciencia, Tecnología e Innovación del Distrito Federal” (SECITI), for the financial support. We address our most sincerely gratefulness to Prof. Jośe Trujillo and Marving Soriano. A. Pulzara Mora thanks to Secretaría de Ciencia, Tecnología e Innovación del Distrito Federal (CECITI-DF) and Centro Latinoamericano de Física (CLAF) for financial support.

\section{References}

[1] Borri, P., Schneider, S., Langbein, W. and Bimberg, D. (2006) Ultrafast Carrier Dynamics in InGaAs Quantum Dot Materials and Devices. Journal of Optics A: Pure and Applied Optics, 84, S33-S46.

[2] Zilkie, A.J., Meier, J., Mojahedi, M., Poole, P.J., et al. (2007) Carrier Dynamics of Quantum-Dot, Quantum-Dash, and Quantum-Well Semiconductor Optical Amplifiers Operating at 1.55 mm. IEEE Journal of Quantum Electronics, 4311, 1873-1880.

[3] Franta, D., Ohlídal, I., Klapetek, P., Montaigne-Ramil, A., Bonanni, A., et al. (2002) Influence of Overlayers on Determination of the Optical Constants of Zn Sethin Films. Journal of Applied Physics, 92, 1873-1880. 
http://dx.doi.org/10.1063/1.1489068

[4] Klapetek, P., Ohlídal, I., Franta, D., Montaigne-Ramil, A., Bonanni, A., et al. (2003) Atomic Force Microscopy Characterization of ZnTe Epitaxial Films. Acta Physical Slovaca, 53, 223-230.

[5] Jenkins, C., Westwood, D.I., Elliott, M., Macdonald, J.E., Meaton, C., et al. (2001) Metrology of Semiconductor Device Structures by Cross-Sectional AFM. Materials Science and Engineering, 80, 138-141.

[6] Fasching, G., Schrey, F.F., Roch, T., Andrews, A.M., Brezna, W., et al. (2006) Single InAs/GaAs Quantum Dots: Photocurrent and Cross-Sectional AFM Analysis. Physica E: Low-Dimensional Systems and Nanostructures, 32, 183-186. http://dx.doi.org/10.1016/j.physe.2005.12.034

[7] Noy, A., Sanders, C.H., Vezenov, D.V., Wong, S.S. and Lieber, C.M. (1998) Chemically-Sensitive Imaging in Tapping Mode by Chemical Force Microscopy: Relationship between Phase Lag and Adhesion. Langmuir, 14, 1508-1511.

[8] García, R., Tamayo, J., Calleja, M. and García, F. (1998) Phase Contrast in Tapping-Mode Scanning Force Microscopy. Applied Physics A, 66, S309-S312.

[9] Xu, W., Wood-Adams, P.M. and Robertson, C.G. (2006) Measuring Local Viscoelastic Properties of Complex Materials with Tapping Mode Atomic Force Microscopy. Polymer, 47, 4798-4810. http://dx.doi.org/10.1016/j.polymer.2006.04.032

[10] Magonov, S.N., Elings, V. and Whangbo, M.H. (1997) Phase Imaging and Stiffness in Tapping-Mode Atomic Force Microscopy. Surface Science, 375, L385-L391.

[11] http://gwyddion.net/

[12] Horcas, I., Fernandez, R., Gomez-Rodriguez, J.M., Colchero, J., Gomez-Herrero, J., et al. (2007) WSXM: A Software for Scanning Probe Microscopy and a Tool for Nanotechnology. Review of Scientific Instruments, 78, Article ID: 013705.

[13] http://nanoscaleworld.bruker-axs.com/nanoscaleworld/media/p/2740.aspx

\section{Appendix}

[001]

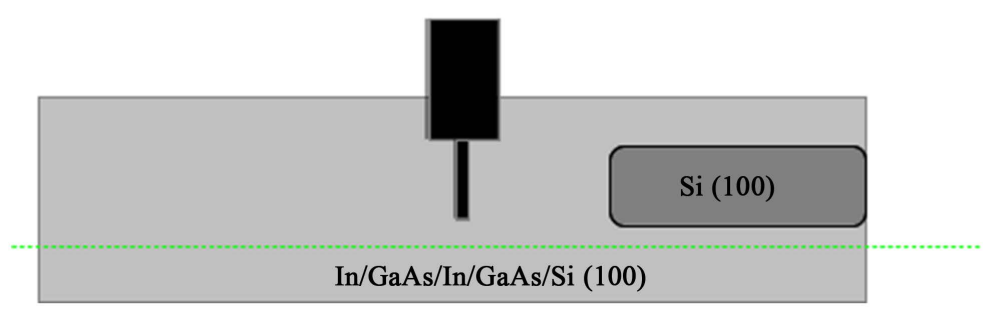

(a)

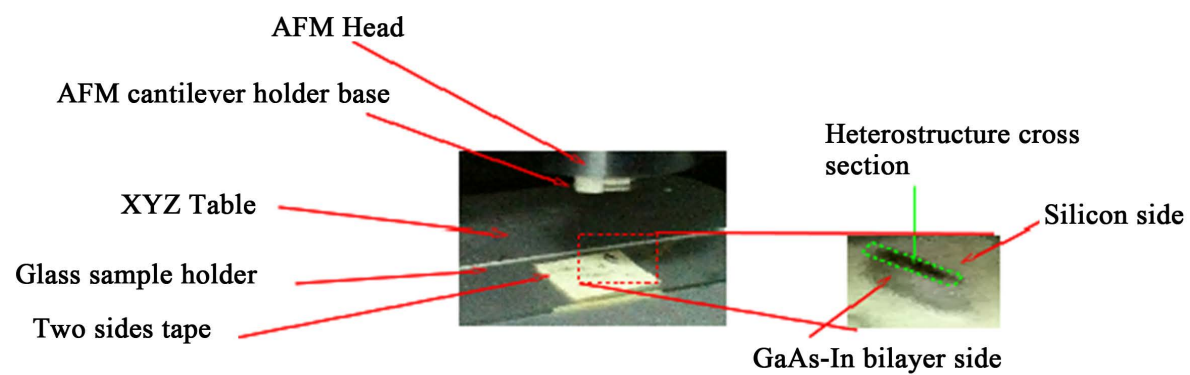

(b) 
Scientific Research Publishing (SCIRP) is one of the largest Open Access journal publishers. It is currently publishing more than 200 open access, online, peer-reviewed journals covering a wide range of academic disciplines. SCIRP serves the worldwide academic communities and contributes to the progress and application of science with its publication.

Other selected journals from SCIRP are listed as below. Submit your manuscript to us via either submit@scirp.org or Online Submission Portal.
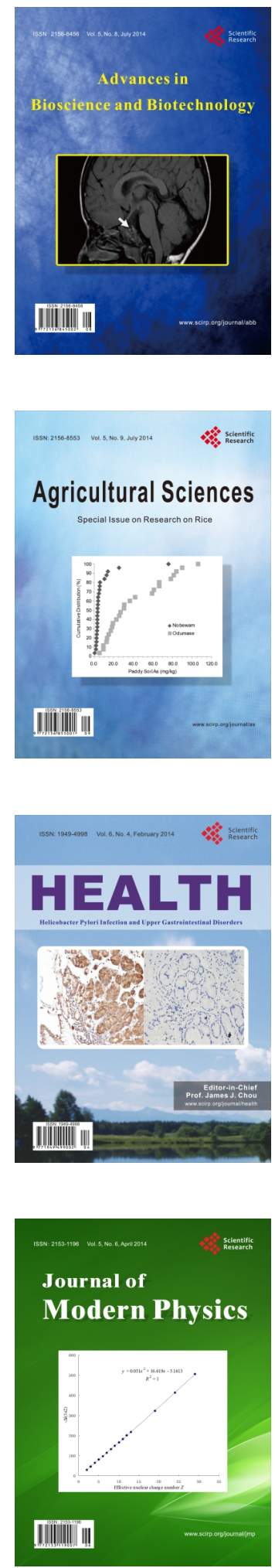
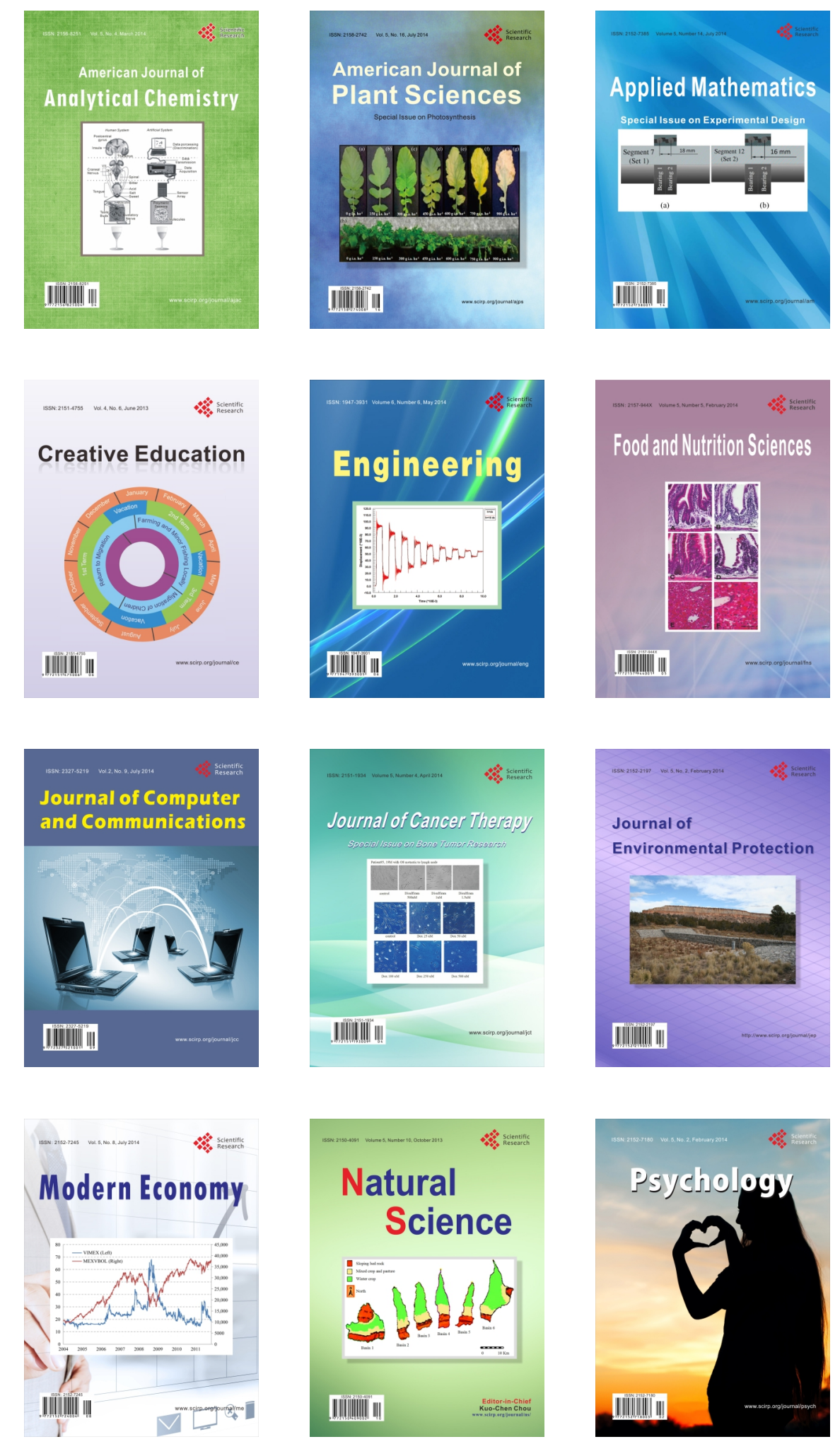